\title{
Synthesis and antimicrobial activity of some novel thienopyrimidines and triazolothienopyrimidines
}

\author{
NITINKUMAR S SHETTY, RAVI S LAMANI and IMTIYAZ AHMED M KHAZI* \\ Department of Chemistry, Karnatak University, Dharwad 580003 \\ e-mail: drimkorgchem@gmail.com
}

MS received 1 July 2008; revised 3 September 2008

\begin{abstract}
Novel tricyclic thienopyrimidines $(3,5,6,9,11,12)$ and triazole fused tetracyclic thienopyrimidines $(\mathbf{4 a}-\mathbf{c}, \mathbf{1 0} \mathbf{a}-\mathbf{c})$ were synthesized from precursors 2-amino-6-methyl-4,5,6,7-tetrahydro-1benzothiophene-3-carbonitrile 1 and 2-amino-7-oxo-4,5,6,7-tetrahydro-1-benzothio-phene-3-carbonitrile 7 respectively. The corresponding precursors were prepared by employing the Gewald reaction. The structures of newly synthesized compounds were characterized by spectral and analytical data. All the compounds were screened for their biological activities. Some of the compounds displayed promising antibacterial and antifungal activities.
\end{abstract}

Keywords. Thienopyrimidine; triazolothienopyrimidine; Gewald reaction; antibacterial activity; antifungal activity.

\section{Introduction}

Since resistance of pathogenic bacteria towards available antibiotics is rapidly becoming a major worldwide problem, the design of new compounds to deal with resistant bacteria has become one of the most important areas of antibacterial research today. In addition, it is known that antifungal drugs do not have selective activity because of the biochemical similarity between human cell and fungi forms. Therefore there are many studies focused on antibacterial and antifungal compounds. ${ }^{1-3}$

Thienopyrimidines and thiadiazolothienopyrimidines have been found to exhibit a variety of biological activities viz. anti inflammatory, ${ }^{4,5}$ antimicrobial, ${ }^{6}$ analgesic ${ }^{7}$ activities, inhibition of cancer cell proliferation, ${ }^{8}$ antagonism of $\alpha_{1}$ adrenoceptors ${ }^{9}$ and prevention of cartilage destruction in articular diseases. ${ }^{10}$ Consequently, thienopyrimidines ${ }^{11,12}$ have become a wellsought-privileged class of compounds in drug discovery programs. In view of these reports and in continuation of our work on biologically active nitrogen and sulfur heterocycles, ${ }^{13-15}$ we report here the synthesis of some novel thienopyrimidines and thienotriazolopyrimidines for the evaluation of their antimicrobial properties. The

\footnotetext{
*For correspondence
}

synthesized compounds were tested against two Gram (+) Bacteria (Staphylococcus aureus, Bacillus subtilis), two Gram (-) bacteria (Pseudomonas aeruginosa, Escherichia coli) and two yeast-like fungi Candida albicans and Candida parapsilosis using the broth microdilution method.

\section{Experimental}

\subsection{General}

Melting points were determined in open capillaries and are uncorrected. The IR spectra were recorded on Nicolet Impact 410 FT IR spectrophotometer using $\mathrm{KBr}$ pellets. ${ }^{1} \mathrm{H}$ and ${ }^{13} \mathrm{C}$ NMR were recorded on Bruker $300-\mathrm{MHz}$ FT NMR spectrometer in $\mathrm{CDCl}_{3}$ and DMSO- $d_{6}$ with TMS as internal standard. Mass spectra were recorded on Finnigan MAT (Model MAT8200) spectrometer and elemental analysis were carried out using Heraus $\mathrm{CHN}$ rapid analyser.

\section{1a 2-Amino-6-methyl-4,5,6,7-tetrahydro-benzo-}

[b]thiophene-3-carbonitrile (1): To a well stirred mixture of 4-methylcyclohexanone ( $8 \mathrm{~g}, 71 \mathrm{mmole})$ and malononitrile $(4.7 \mathrm{~g}, 71 \mathrm{mmole})$ in ethanol $(45 \mathrm{~mL})$ was added elemental sulfur $(2.31 \mathrm{~g}$, 72 mmole). To this cooled reaction mixture was added diethylamine $(5 \mathrm{~mL})$ with vigorous stirring 
during $1 \mathrm{~min}$. Reaction mixture was stirred at 40 $45^{\circ} \mathrm{C}$ for about $1 \mathrm{~h}$. The yellow-orange solid separated was filtered, washed with hot ethanol and recrystallised from dioxane to yield analytically pure yellow needles. ${ }^{16}$ Yield $9.7 \mathrm{~g}$ (71\%); m.p. 138$140^{\circ} \mathrm{C}$.

$2.1 \mathrm{~b} N$-(3-Cyano-6-methyl-4,5,6,7-tetrahydro-benzo [b]thiophene-2-yl)formimidic acid ethyl ester (2): A solution of $1(1.10 \mathrm{~g}, 57 \mathrm{mmole})$ in triethylorthoformate $(12 \mathrm{~mL})$ was heated under reflux for $18 \mathrm{~h}$, excess triethylorthoformate was removed under vaccum. The residue was treated with petroleum ether. Solid that separated was filtered and recrystallized with petroleum ether to afford light brown crystals. Yield 1.18 g (83\%); m.p. $136-138^{\circ} \mathrm{C}$; IR (KBr) $v$ $\mathrm{cm}^{-1} 3090,2921,2866,2212,1625,1573,1541 ;{ }^{1} \mathrm{H}$ NMR $\left(300 \mathrm{MHz}, \mathrm{CDCl}_{3}\right) \delta 1.26(d, 3 \mathrm{H}, J=5.3 \mathrm{~Hz}$, $\left.\mathrm{CH}_{3}\right), 1.41\left(t, J=7.1 \mathrm{~Hz}, 3 \mathrm{H}, \mathrm{CH}_{2} \mathrm{CH}_{3}\right), 2.03(\mathrm{~m}$, $\left.1 \mathrm{H}, \mathrm{C}_{6}-\mathrm{H}\right), 2.22\left(d, J=3.2 \mathrm{~Hz}, 2 \mathrm{H}, \mathrm{CH}_{2}\right), 2.48(t$, $\left.2 \mathrm{H}, \mathrm{CH}_{2}\right), 2.60\left(t, 2 \mathrm{H}, \mathrm{CH}_{2}\right), 4.42(q, J=7.08 \mathrm{~Hz}$, $\left.2 \mathrm{H}, \mathrm{CH}_{2} \mathrm{CH}_{3}\right), 7.94(s, 1 \mathrm{H}, \mathrm{N}=\mathrm{CH}) ;{ }^{13} \mathrm{C} \quad \mathrm{NMR}$ $\left(75 \mathrm{MHz}, \mathrm{CDCl}_{3}\right) \delta 14.7,18.9,19.5,37 \cdot 2,39 \cdot 3$, $41 \cdot 0,59 \cdot 5,112 \cdot 8,115 \cdot 9,133.4,136 \cdot 0,144 \cdot 2,163 \cdot 9$; Anal. calcd. for $\mathrm{C}_{13} \mathrm{H}_{16} \mathrm{~N}_{2} \mathrm{OS}$ : C, 62.87; H, 6.49; N, 11.28. Found: C, 62.76; H, 6.57; N, 11.38.

2.1c 4-Imino-7-methyl-5, 6, 7,8-tetrahydro[1]benzothieno[2,3-d]pyrimidin-3 [4H]-amine (3): A mixture of 2 (1.38 g, $55 \mathrm{mmole})$ and hydrazine hydrate $(7 \mathrm{~mL})$ was stirred at room temperature for $2 \mathrm{~h}$ and then diluted with ethanol $(20 \mathrm{~mL})$. The resulting fine solid suspension was filtered, washed with ethanol and purified by recrystallisation from ethanoldioxane mixture to afford analytically pure pale yellow granules. Yield $1 \mathrm{~g}(77 \%)$; m.p. $152-154^{\circ} \mathrm{C}$; IR (KBr) $v \mathrm{~cm}^{-1} 3382,3271,3180,3037,2929,1614$, 1548; ${ }^{1} \mathrm{H}$ NMR $\left(300 \mathrm{MHz}, \mathrm{DMSO}-d_{6}\right) \delta 1.12(d$, $\left.J=5.2 \mathrm{~Hz}, 3 \mathrm{H}, \mathrm{CH}_{3}\right), 2.15\left(m, \mathrm{C}_{7}-\mathrm{H}, 1 \mathrm{H}\right), 2.32(d$, $\left.2 \mathrm{H}, J=3.2 \mathrm{~Hz}, \mathrm{CH}_{2}\right), 2.38\left(t, 2 \mathrm{H}, \mathrm{CH}_{2}\right), 2.40(t, 2 \mathrm{H}$, $\mathrm{CH}_{2}$ ), 4.72 (brs $, 2 \mathrm{H}, \mathrm{NH}_{2}, \mathrm{D}_{2} \mathrm{O}$ exchangeable), 5.54 (br s, $1 \mathrm{H}, \mathrm{NH}, \mathrm{D}_{2} \mathrm{O}$ exchangeable), $7.96(s, 1 \mathrm{H}$, $\mathrm{C}_{2}-\mathrm{H}$, pyrimidine); ${ }^{13} \mathrm{C}$ NMR $\left(75 \mathrm{MHz}, \mathrm{DMSO}-d_{6}\right)$ $\delta 18 \cdot 2,20 \cdot 1,32 \cdot 5,38 \cdot 0,43 \cdot 9,119 \cdot 8,128 \cdot 2,135 \cdot 0$, 142.4, 162.5, 163.9; Anal. calcd. For $\mathrm{C}_{11} \mathrm{H}_{14} \mathrm{~N}_{4} \mathrm{~S}$ : C, $56 \cdot 38$; H, 6.02; N, 23.91. Found: C, 56.23; H, 6.12; $\mathrm{N}, 23 \cdot 99$.

\section{1d 7-Methyl-5, 6, 7,8-tetrahydro[1]benzothieno} [2,3-d]pyrimidin-4[3H]-one (5): A mixture of 2amino-6-methyl-4,5,6,7-tetrahydro-benzo[b]thiophene- 3-carbonitrile 1 ( $1 \mathrm{~g}, 52 \mathrm{mmole})$ and formic acid $(15 \mathrm{~mL})$ was heated under reflux for $5 \mathrm{~h}$. The excess of formic acid was removed under reduced pressure. The resulting residue was crystallized from ethanol. Yield $0.92 \mathrm{~g}$ (80\%); m.p. $154-156^{\circ} \mathrm{C}$; IR (KBr) $v \mathrm{~cm}^{-1} 3158,3070,1664,1580,1364,974 ;{ }^{1} \mathrm{H}$ NMR $\left(300 \mathrm{MHz}, \mathrm{CDCl}_{3}\right) \delta 1.35\left(d, J=5.3 \mathrm{~Hz}, 3 \mathrm{H}, \mathrm{CH}_{3}\right)$, $2.09\left(m, 1 \mathrm{H}, \mathrm{C}_{T}-\mathrm{H}\right), 2.20\left(d, J=3.2 \mathrm{~Hz}, 2 \mathrm{H}, \mathrm{CH}_{2}\right)$, $2.45\left(t, 2 \mathrm{H}, \mathrm{CH}_{2}\right), 2.76\left(t, 2 \mathrm{H}, \mathrm{CH}_{2}\right), 7.86(s, 1 \mathrm{H}$, $\mathrm{C}_{2}-\mathrm{H}$, pyrimidine), $11.80\left(\right.$ br $s, 1 \mathrm{H}, \mathrm{NH}, \mathrm{D}_{2} \mathrm{O}$ exchangeable); ${ }^{13} \mathrm{C}$ NMR (75 MHz, $\left.\mathrm{CDCl}_{3}\right) \delta 16 \cdot 4,18 \cdot 1$, $31 \cdot 9,38 \cdot 2,42 \cdot 1,130 \cdot 9,136 \cdot 0,142 \cdot 5,143 \cdot 1,161 \cdot 9$, 169.9; Anal. calcd. for $\mathrm{C}_{11} \mathrm{H}_{12} \mathrm{~N}_{2} \mathrm{OS}$ : C, 59.97; $\mathrm{H}$, $5 \cdot 49 ; \mathrm{N}, 12 \cdot 72$. Found: C, 59.89; H, 5.69, N, 12.37.

\section{1e 7-Methyl-5, 6, 7,8-tetrahydro[1]benzothieno}

[2,3-d]pyrimidin-4-amine (6): Imidoformate $\mathbf{2}$ $(1.38 \mathrm{~g}, 55 \mathrm{mmole})$ was treated with anhydrous ethanolic ammonia $(35 \mathrm{~mL})$ at $0^{\circ} \mathrm{C}$. The bright yellow solid that separated within 10 min was further stirred overnight. Solvent was removed in vaccuo and the residue was dissolved in DMF $(35 \mathrm{~mL})$ to which sodium ethoxide $(0.8 \mathrm{~g})$ was added and the stirred solution was heated to $100-112^{\circ} \mathrm{C}$ for $1 \mathrm{~h}$. The solution was concentrated under reduced pressure. The residue was treated with warm water and the product was filtered and recrystallized with aq. ethanol to afford pale yellow granules. Yield $0.70 \mathrm{~g}$ (80\%); m.p. $134-136^{\circ} \mathrm{C}$; IR (KBr) $v \mathrm{~cm}^{-1} 3360$, $3313,3113,1645,1571 ;{ }^{1} \mathrm{H}$ NMR $(300 \mathrm{MHz}$, $\left.\mathrm{CDCl}_{3}\right) \delta 2.01\left(d, J=5.2 \mathrm{~Hz}, 3 \mathrm{H}, \mathrm{CH}_{3}\right), 2.23(\mathrm{~m}$, $\left.1 \mathrm{H}, \mathrm{C}_{7}-\mathrm{H}\right), 2.50\left(d, J=3.1 \mathrm{~Hz}, 2 \mathrm{H}, \mathrm{CH}_{2}\right), 2.85(t$, $\left.2 \mathrm{H}, \mathrm{CH}_{2}\right), 2.96\left(t, 2 \mathrm{H}, \mathrm{CH}_{2}\right), 6.74\left(b r s, 2 \mathrm{H}, \mathrm{NH}_{2}\right.$, $\mathrm{D}_{2} \mathrm{O}$ exchangeable), $8 \cdot 16(s, 1 \mathrm{H}, \mathrm{C} 2-\mathrm{H})$, pyrimidine; ${ }^{13} \mathrm{C}$ NMR $\left(75 \mathrm{MHz}, \mathrm{CDCl}_{3}\right) \delta 20 \cdot 1,20 \cdot 9,30 \cdot 5,38 \cdot 1$, 44.1, 121.2, 129.2, 133.3, 142.0, 157.4, 167.7; Anal. calcd. for $\mathrm{C}_{11} \mathrm{H}_{13} \mathrm{~N}_{3} \mathrm{~S}$ : C, 60.24; H, 5.97; N, 19.16. Found: C, 60.39; H, 5.81, N, 19.28.

\section{1f 9-Methyl-8,9,10,11-tetrahydro[1]benzothieno} [3,2-e][1,2,4]triazolo[1,5-c] pyrimidine (4a): A mixture of $3(0.125 \mathrm{~g}, 0.053 \mathrm{mmole})$, triethylorthoformate $(0.6 \mathrm{~mL})$ and dimethylformamide $(0.6 \mathrm{~mL})$ was heated on water bath $\left(90^{\circ} \mathrm{C}\right)$ for $4 \mathrm{~h}$. The reaction mixture was cooled to room temperature and diluted with petroleum ether $(2 \mathrm{~mL})$. The solid that separated was washed with benzene and petroleum ether and dried. The crude product was purified by column chromatography (EtOAc : benzene :: $6: 4$ ) to furnish yellow granules. Yield $0.09 \mathrm{~g}(70 \%) ; \mathrm{m} . \mathrm{p}$. $122-124^{\circ} \mathrm{C}$; IR (KBr) $v \mathrm{~cm}^{-1} 3076,2949,2921$, 
1620,$1485 ;{ }^{1} \mathrm{H}$ NMR $\left(300 \mathrm{MHz}, \mathrm{CDCl}_{3}\right) \delta 1 \cdot 16(d$, $\left.J=5.4 \mathrm{~Hz}, 3 \mathrm{H}, \mathrm{CH}_{3}\right), 2.09\left(d, J=3.2 \mathrm{~Hz}, 2 \mathrm{H}, \mathrm{CH}_{2}\right)$, $2.12\left(m, \mathrm{C}_{9}-\mathrm{H}, 1 \mathrm{H}\right), 2.58\left(t, 2 \mathrm{H}, \mathrm{CH}_{2}\right), 2.99(t, 2 \mathrm{H}$, $\left.\mathrm{CH}_{2}\right), 8.42\left(s, 1 \mathrm{H}, \mathrm{C}_{2}-\mathrm{H}\right.$, pyrimidine $), 9.20(s, 1 \mathrm{H}$, triazole); ${ }^{13} \mathrm{C}$ NMR $\left(75 \mathrm{MHz}, \mathrm{CDCl}_{3}\right) \delta 18 \cdot 1,19 \cdot 8$, $22 \cdot 2,35 \cdot 6,41 \cdot 9,122 \cdot 4,129 \cdot 3,133 \cdot 6,142 \cdot 7,145 \cdot 9$, 149.1, 157.2; Anal. calcd. for $\mathrm{C}_{12} \mathrm{H}_{12} \mathrm{~N}_{4} \mathrm{~S}$ : C, 58.99; $\mathrm{H}, 4.95 ; \mathrm{N}, 22.93$. Found: C, 59.08; H, 4.88; N, $23 \cdot 03$.

\section{1g 2-Methyl-9-methyl-8,9,10,11-tetrahydro[1]} benzothieno[3,2-e][1,2,4]triazolo[1,5-c] pyrimidine (4b): A mixture of $3(0.125 \mathrm{~g}, 0.053 \mathrm{mmole})$, triethylorthoacetate $(0.6 \mathrm{~mL})$ and dimethylformamide $(0.6 \mathrm{~mL})$ was heated on water bath $\left(90^{\circ} \mathrm{C}\right)$ for $4 \mathrm{~h}$. The reaction mixture was cooled to room temperature and diluted with petroleum ether $(2 \mathrm{~mL})$. The solid that separated was washed with benzene and petroleum ether and dried. The crude product was purified by column chromatography (EtOAc: benzene :: 6:4) to furnish yellow granules. Yield 0.09 (66\%); m.p. $149-151^{\circ} \mathrm{C}$; IR (KBr) $v \mathrm{~cm}^{-1} 3044$, 2953, 2927, 1616, 1555; ${ }^{1} \mathrm{H}$ NMR $(300 \mathrm{MHz}$, $\left.\mathrm{CDCl}_{3}\right) \delta 1.25\left(d, J=5.2 \mathrm{~Hz}, 3 \mathrm{H}, \mathrm{CH}_{3}\right), 2.05(d$, $\left.J=3.1 \mathrm{~Hz}, 2 \mathrm{H}, \mathrm{CH}_{2}\right), 2.20\left(m, 1 \mathrm{H}, \mathrm{C}_{9}-\mathrm{H}\right), 2.43(t$, $\left.2 \mathrm{H}, \mathrm{CH}_{2}\right), 2.81\left(t, 2 \mathrm{H}, \mathrm{CH}_{2}\right), 3.35\left(s, 3 \mathrm{H}, \mathrm{CH}_{3}\right), 8.98$ $\left(s, 1 \mathrm{H}, \mathrm{C}_{2}-\mathrm{H}\right.$, pyrimidine); ${ }^{13} \mathrm{C}$ NMR $(75 \mathrm{MHz}$, $\left.\mathrm{CDCl}_{3}\right) \delta 15 \cdot 4,19 \cdot 9,22 \cdot 4,34 \cdot 5,38 \cdot 0,43 \cdot 1,121 \cdot 1$, 127.6, 133.0, 139.9, 148.0, 156.7, 159.0; Anal. calcd. for $\mathrm{C}_{13} \mathrm{H}_{14} \mathrm{~N}_{4} \mathrm{~S}: \mathrm{C}, 60.44 ; \mathrm{H}, 5.46 ; \mathrm{N}, 21.69$. Found: C, $60.59 ; \mathrm{H}, 5 \cdot 38 ; \mathrm{N}, 21 \cdot 75$.

2.1h 2-Ethyl-9-methyl-8,9,10,11-tetrahydro[1]benzothieno[3,2-e][1,2,4]triazolo[1,5-c]pyrimidine (4c): Prepared as per the procedure mentioned for compound $4 \mathbf{a}$ and $\mathbf{4 b}$. In this case triethylorthopropionate was used as cyclizing agent. yield $0.11 \mathrm{~g}(76 \%)$; pale yellow granules; m.p. $136-138^{\circ} \mathrm{C}$; IR $(\mathrm{KBr})$ $v \mathrm{~cm}^{-1} 3051,2967,2911,1616,1573 ;{ }^{1} \mathrm{H}$ NMR $\left(300 \mathrm{MHz}, \mathrm{CDCl}_{3}\right) \delta 1 \cdot 13\left(d, J=5 \cdot 3 \mathrm{~Hz}, 3 \mathrm{H}, \mathrm{CH}_{3}\right)$, $1.44\left(t, J=6.6 \mathrm{~Hz}, 3 \mathrm{H}, \mathrm{CH}_{2} \mathrm{CH}_{3}\right), 2.07\left(m, 1 \mathrm{H}, \mathrm{C}_{9}\right.$ $\mathrm{H}, 1 \mathrm{H}), 2.11\left(d, J=3.2 \mathrm{~Hz}, 2 \mathrm{H}, \mathrm{CH}_{2}\right), 2.62(t, 2 \mathrm{H}$, $\left.\mathrm{CH}_{2}\right), 2.85\left(t, 2 \mathrm{H}, \mathrm{CH}_{2}\right), 4.48(q, J=7.02 \mathrm{~Hz}, 2 \mathrm{H}$, $\left.\underline{\mathrm{CH}_{2}} \mathrm{CH}_{3}\right), 8.90\left(s, 1 \mathrm{H}, \mathrm{C}_{2}-\mathrm{H}\right.$, pyrimidine); ${ }^{13} \mathrm{C}$ NMR $\left(75 \mathrm{MHz}, \mathrm{CDCl}_{3}\right) \delta 15 \cdot 9,19 \cdot 3,22 \cdot 6,24 \cdot 3,33 \cdot 1$, $36 \cdot 5,41 \cdot 9,122 \cdot 0,127 \cdot 3,135 \cdot 1,140 \cdot 6,145 \cdot 9,157 \cdot 2$, 160.0; Anal. calcd. for $\mathrm{C}_{14} \mathrm{H}_{16} \mathrm{~N}_{4} \mathrm{~S}$ : C, 61.74; $\mathrm{H}$, $5 \cdot 92 ; \mathrm{N}, 20 \cdot 57$. Found: C, 61.62; H, 5.85; N, 20.64.

2.1i 2-Amino-7-oxo-4,5, 6,7-tetrahydro-1-benzothiophene-3-carbonitrile (7): To a well stirred mixture of 1,3-cyclohexanedione $(8 \cdot 2 \mathrm{~g}, 73 \mathrm{mmole})$ and malononitrile $(4.8 \mathrm{~g}, 73 \mathrm{mmole})$ in ethanol $(45 \mathrm{~mL})$ was added elemental sulfur $(2.3 \mathrm{~g}, 72 \mathrm{mmole})$. To this cooled reaction mixture was added diethylamine $(5 \mathrm{~mL})$ with vigorous stirring during $1 \mathrm{~min}$. Reaction mixture was stirred at $40-45^{\circ} \mathrm{C}$ for about $1 \mathrm{~h}$. The yellow-orange solid separated was filtered, washed with hot ethanol and recrystallised from dioxane to yield analytically pure yellow needles. Yield $9.8 \mathrm{~g}$ (70\%); m.p. $215-217^{\circ} \mathrm{C}$; IR (KBr) $v \mathrm{~cm}^{-1} 3339$, $3190,2962,2901,2213,1645,1623 ;{ }^{1} \mathrm{H}$ NMR $\left(300 \mathrm{MHz}, \mathrm{DMSO}-d_{6}\right) \delta 2 \cdot 27\left(t, 2 \mathrm{H}, \mathrm{CH}_{2}\right), 2.50(m$, $\left.2 \mathrm{H}, \mathrm{CH}_{2}\right), 2.72\left(t, 2 \mathrm{H}, \mathrm{CH}_{2}\right), 8.30\left(s, 2 \mathrm{H}, \mathrm{NH}_{2}, \mathrm{D}_{2} \mathrm{O}\right.$ exchangeable); ${ }^{13} \mathrm{C}$ NMR $\left(75 \mathrm{MHz}\right.$, DMSO- $\left.d_{6}\right) \delta$ $19 \cdot 1,23 \cdot 5,39 \cdot 8,115 \cdot 3,116 \cdot 2,142 \cdot 0,144 \cdot 8,148 \cdot 1$, 188.2; Anal. calcd. for $\mathrm{C}_{9} \mathrm{H}_{8} \mathrm{~N}_{2} 0 \mathrm{~S}$ : C, $56 \cdot 23 ; \mathrm{H}, 4 \cdot 19$; $\mathrm{N}, 14 \cdot 57$. Found: C, 56.36; H, 4.05; N, 14.63.

\section{1j N-(3-Cyano-7-oxo-4,5,6,7-tetrahydro-benzo[b]} thiophene-2-yl)-formimidic acid ethyl ester (8): A solution of $7(1.10 \mathrm{~g}, 57 \mathrm{mmole})$ in triethylorthoformate $(12 \mathrm{~mL})$ was heated under reflux for $18 \mathrm{~h}$, excess triethylorthoformate was removed under vaccum. The residue was treated with petroleum ether. Solid that separated was filtered and recrystallized with petroleum ether to afford light brown crystals. Yield $0.92 \mathrm{~g}(65 \%)$; m.p. $97-99^{\circ} \mathrm{C}$; IR (KBr) $v \mathrm{~cm}^{-1}$ 2989, 2932, 2882, 2219, 1663, 1573, 1541; ${ }^{1} \mathrm{H}$ NMR $\left(300 \mathrm{MHz}, \mathrm{CDCl}_{3}\right) \delta 1.43 \quad(t, \quad J=6.9 \mathrm{~Hz}, \quad 3 \mathrm{H}$, $\left.\mathrm{CH}_{2} \mathrm{CH}_{3}\right), 2.60\left(t, 2 \mathrm{H}, \mathrm{CH}_{2}\right), 2.89\left(m, 2 \mathrm{H}, \mathrm{CH}_{2}\right), 2.93$ $\left(t, 2 \mathrm{H}, \mathrm{CH}_{2}\right), 4.49\left(q, J=7.09 \mathrm{~Hz}, 2 \mathrm{H}, \mathrm{CH}_{2} \mathrm{CH}_{3}\right)$, $8.04(s, 1 \mathrm{H}, \mathrm{N}=\mathrm{CH}) ;{ }^{13} \mathrm{C}$ NMR $\left(75 \mathrm{MHz}, \mathrm{CDCl}_{3}\right) \delta$ $14 \cdot 1,22 \cdot 9,25 \cdot 1,39 \cdot 8,58 \cdot 3,114 \cdot 6,116 \cdot 4,143 \cdot 2$, 146.0, 148.5, 164.1, 188.6; Anal. calcd. for $\mathrm{C}_{12} \mathrm{H}_{12} \mathrm{~N}_{2} \mathrm{O}_{2} \mathrm{~S}: \mathrm{C}, 58.05 ; \mathrm{H}, 4.87 ; \mathrm{N}, 11.28$. Found: C, 57.93; H, 4.97; N, 11.39.

\section{$2.1 \mathrm{k}$ 3-Amino-4-imino-5,6,7,8-tetrahydro-5H-benzo}

[4,5]thieno[2,3-d]pyrimidin-8-one (9): Prepared from compound $\mathbf{8}$ as per the procedure mentioned for preparation of 3. Yield $0.83 \mathrm{~g}(64 \%)$; m.p. 176$178^{\circ} \mathrm{C}$; light brown crystals; IR $(\mathrm{KBr}) v \mathrm{~cm}^{-1} 3406$, $3348,3235,3037,2935,1664,1582 ;{ }^{1} \mathrm{H}$ NMR $\left(300 \mathrm{MHz}, \mathrm{DMSO}-d_{6}\right) \delta 2.39\left(t, 2 \mathrm{H}, \mathrm{CH}_{2}\right), 2.50(\mathrm{~m}$, $\left.2 \mathrm{H}, \mathrm{CH}_{2}\right), 2.90\left(t, 2 \mathrm{H}, \mathrm{CH}_{2}\right), 4.54\left(b r s, 2 \mathrm{H}, \mathrm{NH}_{2}\right.$, $\mathrm{D}_{2} \mathrm{O}$ exchangeable), 5.31 (br s, 1H, NH, $\mathrm{D}_{2} \mathrm{O}$ exchangeable $), 7.46\left(s, 1 \mathrm{H}, \mathrm{C}_{2}-\mathrm{H}\right.$, pyrimidine $) ;{ }^{13} \mathrm{C}$ NMR $\left(75 \mathrm{MHz}\right.$, DMSO- $\left.d_{6}\right) \delta 19.2,24 \cdot 0,38.9$, $130 \cdot 1,133 \cdot 7,140 \cdot 5,145 \cdot 7,164 \cdot 1,165 \cdot 2,190 \cdot 8$; Anal. calcd. for $\mathrm{C}_{10} \mathrm{H}_{10} \mathrm{~N}_{4} \mathrm{OS}: \mathrm{C}, 51.27 ; \mathrm{H}, 4.30 ; \mathrm{N}$, 23.91. Found: C, 51.36; H, 4.39; N, 23.82. 
2.11 6,7-Dihydro-[1]benzothieno[2,3-d]pyrimidin4,8(3H,5H)-dione (11): Prepared from compound 7 as per the procedure mentioned for preparation of 5. Yield $0.92 \mathrm{~g}(80 \%)$; pale yellow granules; m.p. $154-156^{\circ} \mathrm{C}$; IR $(\mathrm{KBr}) v \mathrm{~cm}^{-1} 3087,2955,1672$, $1585,1375,990 ;{ }^{1} \mathrm{H}$ NMR $\left(300 \mathrm{MHz}, \mathrm{CDCl}_{3}\right) \delta 2.45$ $\left(t, 2 \mathrm{H}, \mathrm{CH}_{2}\right), 2.72\left(m, 2 \mathrm{H}, \mathrm{CH}_{2}\right), 2.93\left(t, 2 \mathrm{H}, \mathrm{CH}_{2}\right)$, $7.51\left(s, 1 \mathrm{H}, \mathrm{C}_{2}-\mathrm{H}\right.$, pyrimidine), $11.98(\mathrm{br} s, 1 \mathrm{H}, \mathrm{NH}$, $\mathrm{D}_{2} \mathrm{O}$ exchangeable); ${ }^{13} \mathrm{C}$ NMR $\left(75 \mathrm{MHz}, \mathrm{CDCl}_{3}\right) \delta$ $18 \cdot 2,23 \cdot 8,42 \cdot 3,136 \cdot 0,139 \cdot 2,145 \cdot 1,147 \cdot 4,164 \cdot 3$, 173.8, 189.0; Anal. calcd. for $\mathrm{C}_{10} \mathrm{H}_{8} \mathrm{~N}_{2} \mathrm{O}_{2} \mathrm{~S}$ : C, 54.53 ; H, 3.66; N, 12.72. Found: C, 54.41; H, 3.55; $\mathrm{N}, 12 \cdot 86$.

\section{$2.1 \mathrm{~m}$ 4-Amino-6,7-dihydro[1]benzothieno [2,3-d]} pyrimidin-8(5H)-one (12): Prepared from compound 8 as per the procedure mentioned for preparation of 6 . Yield $0.91 \mathrm{~g}(75 \%)$; pale yellow granules; m.p. $143-145^{\circ} \mathrm{C}$; IR (KBr) $v \mathrm{~cm}^{-1} 3360,3313,3113$, 1645,$1571 ;{ }^{1} \mathrm{H}$ NMR $\left(300 \mathrm{MHz}, \mathrm{CDCl}_{3}\right) \delta 2.50(t$, $\left.2 \mathrm{H}, \mathrm{CH}_{2}\right), 2.85\left(m, 2 \mathrm{H}, \mathrm{CH}_{2}\right), 2.96\left(t, 2 \mathrm{H}, \mathrm{CH}_{2}\right)$, $6.74\left(b r s, 2 \mathrm{H}, \mathrm{NH}_{2}, \mathrm{D}_{2} \mathrm{O}\right.$ exchangeable), $8.16(s$, $1 \mathrm{H}, \mathrm{C}_{2}-\mathrm{H}$, pyrimidine); ${ }^{13} \mathrm{C}$ NMR $\left(75 \mathrm{MHz}, \mathrm{CDCl}_{3}\right.$ ) $\delta 24 \cdot 2,25 \cdot 0,38 \cdot 9,132 \cdot 0,135 \cdot 1,140 \cdot 2,142 \cdot 1,156 \cdot 3$, 166.8, 190.1; Anal. calcd. for $\mathrm{C}_{10} \mathrm{H}_{9} \mathrm{~N}_{3} \mathrm{OS}$ : C, 54.68; H, 4.06; N, 19.23. Found: C, 54.54; H, 3.96; N, $19 \cdot 32$.

\section{1n 8,9,10,11-Tetrahydro[1]benzothieno[3,2-e]} [1,2,4]triazolo[1,5-c]pyrimidine-8-one (10a): Prepared from compound 9 as per the procedure mentioned for preparation of 4 . Yield $0.09 \mathrm{~g}(70 \%)$; intense yellow granules; m.p. $182-184^{\circ} \mathrm{C}$; IR $(\mathrm{KBr})$ $v \mathrm{~cm}^{-1} 3045,2949,1658,1617,1522,1485,1432$; ${ }^{1} \mathrm{H}$ NMR $\left(300 \mathrm{MHz}, \mathrm{CDCl}_{3}\right) \delta 2.79\left(t, 2 \mathrm{H}, \mathrm{CH}_{2}\right)$, $2.83\left(m, 2 \mathrm{H}, \mathrm{CH}_{2}\right), 2.97\left(t, 2 \mathrm{H}, \mathrm{CH}_{2}\right), 8.48(s, 1 \mathrm{H}$, $\mathrm{C}_{2}-\mathrm{H}$, pyrimidine $), 9.33(s, 1 \mathrm{H}$, triazole $) ;{ }^{13} \mathrm{C}$ NMR $\left(75 \mathrm{MHz}, \mathrm{CDCl}_{3}\right) \delta 22 \cdot 4,24 \cdot 8,39 \cdot 6,127 \cdot 2,133.9$, $140 \cdot 7,142 \cdot 3,146 \cdot 0,147 \cdot 8,157 \cdot 3,190 \cdot 1$; Anal. calcd. for $\mathrm{C}_{11} \mathrm{H}_{8} \mathrm{~N}_{4} \mathrm{OS}: \mathrm{C}, 54.09 ; \mathrm{H}, 3 \cdot 30 ; \mathrm{N}, 22.94$. Found: C, 53.91; H, 3.40; N, 23.02.

2.1o 2-Methyl-8,9,10,11-tetrahydro[1]benzothieno [3,2-e][1,2,4]triazolo[1,5-c]pyrimidine-8-one (10b): Prepared from compound 9 as per the procedure mentioned for preparation of 4 . Yield $0.1 \mathrm{~g}(73 \%)$; intense yellow granules; m.p. $175-177^{\circ} \mathrm{C}$; IR $(\mathrm{KBr})$ $v \mathrm{~cm}^{-1} 2999,2927,1672,1618,1543 ;{ }^{1} \mathrm{H}$ NMR $\left(300 \mathrm{MHz}, \mathrm{CDCl}_{3}\right) \delta 2.72\left(t, 2 \mathrm{H}, \mathrm{CH}_{2}\right), 2.76(\mathrm{~m}, 2 \mathrm{H}$, $\left.\mathrm{CH}_{2}\right), 3.04\left(t, 2 \mathrm{H}, \mathrm{CH}_{2}\right), 3.77\left(s, 3 \mathrm{H}, \mathrm{CH}_{3}\right), 8.00(s$, $1 \mathrm{H}, \mathrm{C}_{2}-\mathrm{H}$, pyrimidine); ${ }^{13} \mathrm{C}$ NMR $\left(75 \mathrm{MHz}, \mathrm{CDCl}_{3}\right)$ $\delta 15 \cdot 2,22 \cdot 8,25 \cdot 3,39 \cdot 8,131 \cdot 6,134 \cdot 9,141 \cdot 2,142 \cdot 9$, 146.4, 157.2, 162.2, 190.6; Anal. calcd. for $\mathrm{C}_{12} \mathrm{H}_{10} \mathrm{~N}_{4} \mathrm{OS}$ : C, 55.80; H, 3.90; N, 21.69. Found: C, $55 \cdot 71 ; \mathrm{H}, 3 \cdot 96 ; \mathrm{N}, 21 \cdot 78$.

\section{1p 2-Ethyl-8,9,10,11-tetrahydro[1]benzothieno} $[3,2-e][1,2,4]$ triazolo[1,5-c]pyrimidine-8-one (10c): Prepared from compound 9 as per the procedure mentioned for preparation of 4 . Yield $0.1 \mathrm{~g}(69 \%)$; intense yellow granules; m.p. $179-181^{\circ} \mathrm{C}$; IR $(\mathrm{KBr})$ $v \mathrm{~cm}^{-1} 2985,2943,2881,1663,1617,1542 ;{ }^{1} \mathrm{H}$ NMR $\left(300 \mathrm{MHz}, \mathrm{CDCl}_{3}\right) \delta 1.35(t, J=6.6 \mathrm{~Hz}, 3 \mathrm{H}$, $\left.\mathrm{CH}_{2} \mathrm{CH}_{3}\right), 2.72\left(t, 2 \mathrm{H}, \mathrm{CH}_{2}\right), 2.79\left(m, 2 \mathrm{H}, \mathrm{CH}_{2}\right), 2.89$ $\left(t, 2 \mathrm{H}, \mathrm{CH}_{2}\right), 4.23\left(q, J=7.02 \mathrm{~Hz}, 2 \mathrm{H}, \mathrm{CH}_{2} \mathrm{CH}_{3}\right)$, $8.02\left(s, 1 \mathrm{H}, \mathrm{C}_{2}-\mathrm{H}\right.$, pyrimidine); ${ }^{13} \mathrm{C}$ NMR $(75 \mathrm{MHz}$, $\left.\mathrm{CDCl}_{3}\right) \delta 15 \cdot 9,22 \cdot 4,24 \cdot 9,26 \cdot 3,39 \cdot 9,128 \cdot 8,134 \cdot 0$, 139.4, 144.2, 148.0, 157.1, 160.6, 191.1; Anal. calcd. for $\mathrm{C}_{13} \mathrm{H}_{12} \mathrm{~N}_{4} \mathrm{OS}$ : C, 57.34; H, 4.44; N, 20.57. Found: C, 57.25; H, 4.49; N, 20.62.

\section{Results and discussion}

In the present investigation we have synthesized novel fused tricyclic and tetracyclic thienopyrimidines and thienotriazolopyrimidines from precursors 2-amino-6-methyl-4,5,6,7-tetrahydro-1-benzothiophene3 -carbonitrile and 2-amino-7-oxo-4,5,6,7-tetrahydro-1-benzothiophene-3-carbonitrile respectively. The reaction sequences employed for the synthesis of title compounds is shown in scheme 1 . We envisage $\alpha$-aminocarbonitriles ${ }^{17,18}$ as general precursors for the synthesis of broad range of biologically active thienopyrimidines and triazolothienopyrimidines. 2-Amino-6-methyl-4,5,6,7-tetrahydro-1benzothiophene-3-carbonitrile 1 was prepared by Gewald reaction as reported in the literature. ${ }^{18}$ Similarly, 2-amino-7-oxo-4,5,6,7-tetrahydro-1-benzothiophene-3-carbonitrile 7 was prepared by the reaction of 1,3-cyclohexanedione under conditions reported by K. Gewald. ${ }^{19}$ Formation of thiophene having $\alpha$ aminonitrile was characterized by the presence of band at $2210 \mathrm{~cm}^{-1}$ due to cyano group and $\mathrm{N}-\mathrm{H}$ stretching bands at 3339 and $3190 \mathrm{~cm}^{-1}$. Further it was also supported by the presence of $\mathrm{D}_{2} \mathrm{O}$ exchangeable broad singlet at $\delta 7.48$ in ${ }^{1} \mathrm{H}$ NMR spectrum due to $\mathrm{NH}_{2}$ group.

Imidoformates $(\mathbf{2}, \mathbf{8})$ were prepared in excellent yield by treating $(1,7)$ with triethylorthoformate in refluxing temperature. The structure of $(\mathbf{2}, \mathbf{8})$ was assigned by the absence of $\nu_{\mathrm{N}-\mathrm{H}}$ in IR and the presence of a triplet at $\delta 1.3$ and a quartet at 3.99 corre- 


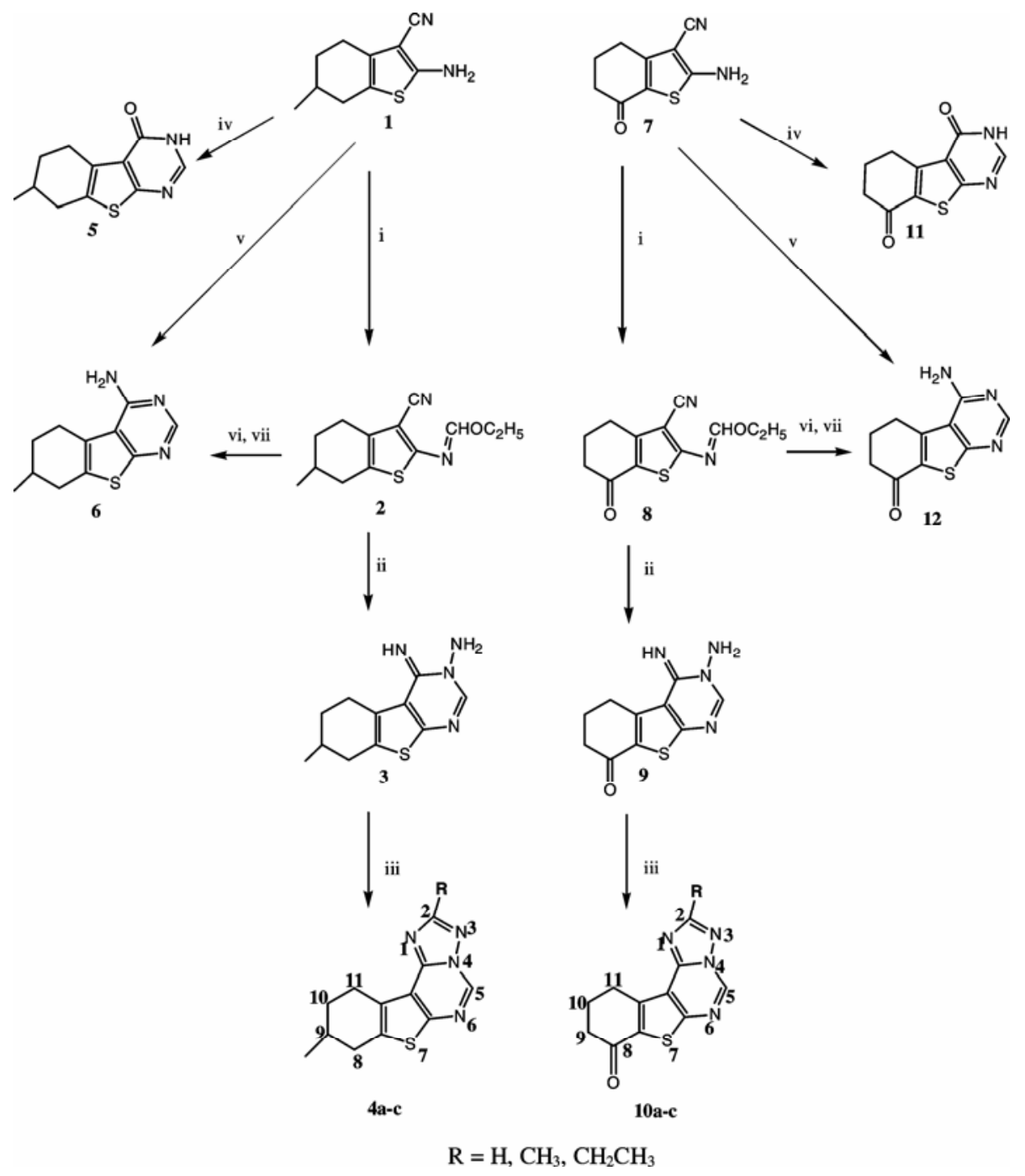

Reagents and conditions: i, Triethylorthoformate, reflux; ii, $\mathrm{NH}_{2} \mathrm{NH}_{2} \cdot \mathrm{H}_{2} \mathrm{O}$, room temp; iii, $\mathrm{RC}(\mathrm{OEt})_{3}, \mathrm{DMF}, 90^{\circ} \mathrm{C}$; iv, $\mathrm{HCOOH}$, reflux; v, $\mathrm{HCONH}_{2}$, reflux; vi, anhydrous ethanolic $\mathrm{NH}_{3}, \mathrm{rt}$; vii, $\mathrm{NaOEt}$ in DMF, $100-112^{\circ} \mathrm{C}$.

\section{Scheme 1.}

sponding to protons of the ethoxy group and peak around $\delta 8.01$ due to $\mathrm{N}=\mathrm{CH}$ in the ${ }^{1} \mathrm{H}-\mathrm{NMR}$ spectrum, along with the other expected signals.

Reactions of imidoformates $(\mathbf{2}, \mathbf{8})$ with hydrazine hydrate afforded the thienopyrimidine $(3,9)$. Formation of the products was confirmed by the presence of bands at 3420,3349 and $3246 \mathrm{~cm}^{-1}$ in IR spectrum, due to amino and imino functional groups. ${ }^{1} \mathrm{H}-$ NMR spectrum shows $\mathrm{D}_{2} \mathrm{O}$ exchangeable singlets at $\delta 4.72$ and 4.35 due to amino and imino groups respectively and the $\mathrm{C}_{2}-\mathrm{H}$ of pyrimidine resonated at $\delta$ 8.15 as a singlet along with other expected signals.

Similarly the reaction of imidoformates $(\mathbf{2}, \mathbf{8})$ with ethanolic ammonia followed by the cyclisation of intermediate with sodium ethoxide in dimethyl formamide resulted in the formation of aminothienopyrimidine $(6,12)$. Formation of the product was established by the presence of characteristic band at $3324,3231 \mathrm{~cm}^{-1}$ due to amino group in IR. The ${ }^{1} \mathrm{H}$ NMR spectrum showed the presence of $\mathrm{D}_{2} \mathrm{O}$ exchangeable broad singlet at $\delta 5.6$ due to $\mathrm{NH}_{2}$ group and the $\mathrm{C}_{2}-\mathrm{H}$ of pyrimidine at $\delta 8.5$ as a singlet. Alternatively aminothienopyrimidines $(\mathbf{6}, \mathbf{1 2})$ were also prepared directly from the corresponding 2-amino-3cyanothiophenes $(1,7)$ precursors by their reaction with formamide. The compounds $(5,11)$ were prepared by refluxing 2-amino-3-cyanothiophenes (1, 7) with formic acid.

The compounds $(\mathbf{3}, 9)$ were further converted into triazolopyrimidine derivatives $(4,10)$ by treatment 
Table 1. Antibacterial and antifungal activities of the compounds as MIC values $(\mu \mathrm{g} / \mathrm{mL})$.

\begin{tabular}{lcccccc}
\hline Compounds & $\begin{array}{c}\text { Staphylococcus } \\
\text { aureus } \\
\text { ATCC } 25923\end{array}$ & $\begin{array}{c}\text { Bacillus } \\
\text { subtilis } \\
\text { ATCC } 6633\end{array}$ & $\begin{array}{c}\text { Escherichia } \\
\text { coli } \\
\text { ATCC 25922 }\end{array}$ & $\begin{array}{c}\text { Pseudomonas } \\
\text { aeruginosa } \\
\text { ATCC 27853 }\end{array}$ & $\begin{array}{c}\text { Candida } \\
\text { albicans } \\
\text { ATCC 10231 }\end{array}$ & $\begin{array}{c}\text { Candida } \\
\text { parapsilosis } \\
\text { ATCC 90018 }\end{array}$ \\
\hline $\mathbf{3}$ & 512 & 128 & 256 & 256 & 32 & 128 \\
$\mathbf{5}$ & 512 & 128 & 256 & 256 & 64 & 64 \\
$\mathbf{6}$ & 256 & 11 & 128 & 256 & 128 & 64 \\
$\mathbf{9}$ & 512 & 256 & 256 & 256 & 16 & 128 \\
$\mathbf{1 1}$ & 512 & 11 & 256 & 256 & 128 & 256 \\
$\mathbf{1 2}$ & 256 & 128 & 256 & 256 & 128 & 64 \\
$\mathbf{4 a}$ & 256 & 64 & 256 & 256 & 32 & 64 \\
$\mathbf{4 b}$ & 128 & 128 & 256 & 256 & 64 & 128 \\
$\mathbf{4 c}$ & 128 & 128 & 256 & 128 & 128 & 128 \\
$\mathbf{1 0 a}$ & 128 & 11 & 128 & 256 & 128 & 64 \\
$\mathbf{1 0 b}$ & 256 & 11 & 128 & 256 & 256 & 64 \\
$\mathbf{1 0 c}$ & 128 & 11 & 256 & 128 & 128 & - \\
Ampicillin & 4 & 8 & 4 & - & - & $0 \cdot 25$ \\
Fluconazole & - & - & - & - & 8 & \\
\hline
\end{tabular}

with triethylorthoesters in dimethylformamide. The formation of triazole ring involving both amino and imino groups was evident by the absence of absorption bands due to either of these groups in the IR spectrum of $(4,10)$. Further ${ }^{1} \mathrm{H}$ NMR spectrum also exhibited the presence of two characteristic protons each as singlet at $\delta 8.4$ and $\delta 9.2$ due to pyrimidine and triazole proton respectively.

All the compounds were evaluated for their antimicrobial properties. MIC's were recorded as the minimum concentration of compound, which inhibits the growth of tested microorganisms. As shown in table 1 , none of the title compounds exhibited any activity against $S$. aureus, $P$. aeruginosa and $E$. coli, but, some of the synthesised compounds were found to be active against $B$. subtilis. The antibacterial activity of compounds $6,11,10 a, 10 b$ and 10c was comparable with that of ampicillin against $B$. subtilis. Hence from the results it can be concluded that the tricyclic aminothienopyrimidines exhibited promising antibacterial activity, while the tetracyclic triazole fused thienopyrimidines also displayed good antibacterial activity against $B$. subtilis. The antifungal activity of compound 9 was $50 \%$ of that of fluconazole against $C$. albicans. Whereas compounds 3 and 4a displayed moderate antifungal activity.

\section{Biological activity}

\subsection{Antimicrobial studies}

Minimum inhibitory concentration (MIC) values for the synthesized compounds were determined by using the broth microdilution method. ${ }^{20,21}$ Two Grampositive ( $S$. aureus ATCC 25923 and B. subtilis ATCC 6633) and two Gram-negative (E. coli ATCC 25922, P. aeruginosa ATCC 27853) bacteria were used as quality control strains. For determining antiyeast activities of the compounds, the following reference strains were tested: Candida albicans ATCC 10231 and Candida parapsilosis ATCC 90018. Ampicillin trihydrate and fluconazole were used as standard antibacterial and antifungal agents, respectively. Fluconazole was dissolved in sterile distilled water, ampicillin trihydrate in phosphate buffer $(\mathrm{pH}$ 8 ) and the stock solution of the synthesized compounds was dissolved in dimethyl sulfoxide (DMSO) and distilled water (50\%) at a concentration of $2048 \mu \mathrm{g} / \mathrm{mL}$. Two fold dilutions of the synthesized compounds were prepared $(1024,512 \ldots \ldots$ $2 \mu \mathrm{g} / \mathrm{mL}$ ), and two fold dilutions of the reference compounds were prepared at $64-0.125 \mu \mathrm{g} / \mathrm{mL}$. All bacteria were cultivated in Mueller-Hinton Agar (Merck). The bacteria inoculums were prepared in Mueller-Hinton Broth (Merck) which had been kept at $36^{\circ} \mathrm{C}$ overnight and was diluted with broth to give a final concentration of $5 \times 10^{5} \mathrm{cfu} / \mathrm{mL}$. All fungi were cultivated in sabouraud Dextrose Agar (Merck). The fungi inculums were prepared in sabouraud liquid medium (oxoid) which had been kept at $36^{\circ} \mathrm{C}$ overnight and was diluted with RPMI1640 medium with L-glutamine buffered with 3-[Nmorpholino]-propane sulfonic acid (MOPS) at $\mathrm{pH} 7$ to give a final concentration of $2.5 \times 10^{3} \mathrm{cfu} / \mathrm{mL}$. The microplates were incubated at $36^{\circ} \mathrm{C}$ and read visually after $24 \mathrm{~h}$, except for candida species when 
it was at $48 \mathrm{~h}$. The incubation chamber was kept humid. At the end of the incubation period, MIC values were recorded as the lowest concentrations of the substances that gave no visible turbidity. The DMSO diluents at a maximum final concentration of $12.5 \%$ had no effect on the microorganisms growth. The minimum inhibitory concentrations (MIC) were noted (table 1).

\section{Conclusion}

The present study reports the synthesis of novel fused tricyclic and tetracyclic thienopyrimidines and thienotriazolopyrimidines from the corresponding precursors 2-amino-6-methyl-4,5,6,7-tetrahydro-1benzothiophene-3-carbonitrile and 2-amino-7-oxo4,5,6,7-tetrahydro-1-benzothio-phene-3-carbonitrile respectively. The investigation of antibacterial screening reveals that the compounds 6, 11, 10a, $10 \mathrm{~b}$ and 10c have exhibited good antibacterial activity against $B$. subtilis comparable to the standard ampicilin, while compounds 3,9 and 4a displayed better antifungal activity against Candida albicans comparable to the standard fluconazole.

\section{References}

1. Gold H S and Moellering R C 1996 Engl. N. J. Med. 3351445

2. Bossche V H, Marichal P and Odds F C 1994 Trends Microbial. 2393

3. Cohen M L 1992 Science 2571050

4. El-Kerdawy M M, Yousif M Y, El-Emam A A, Moustafa M A and El-Sherbeny M A 1996 Boll. Chim. Farmaceutico. 135301

5. Modica M, Santagati M, Santagati A, Cutuli V, Mangano N and Caruso A 2000 Pharmazie 55500
6. Chambhare R V, Khadse B G, Bobde A S and Bahekar R H 2003 Eur. J. Med. Chem. 3889

7. Santagati N A, Caruso A, Cutuli V M and Caccamo F 1995 Il farmaco $\mathbf{5 0} 689$

8. Jennings L D, Kincaid S L, Wang Y D, Krishnamurthy G, Beyer C F, Mginnis J P, Miranda M, Discafani C M and Rabindran S K 2005 Bioorg. Med. Chem. Lett. 154731

9. Meyer M D, Altenbach R J, Basha F Z, Carroll W A, Condon S, Elmore S W, Kerwin J F, Sippy K B, Tietje K, Wendt M D, Hancock A A, Brune M E, Buckner S A and Drizin I $2000 \mathrm{~J}$. Med. Chem. 43 1586

10. Panico A, Cardile V, Santagati A and Gentile B 2001 Il farmaco 56959

11. Vorobev E V, Kletskii M E, Krasnikov V V, Mezheritskii V V and Steglenko D V 2006 Russ. Chem. Bull. $\mathbf{5 5} 2247$

12. Shishoo C J, Devani M B, Ullas G V, Ananthan S and Bhadthi V S 1981 J. Het. Chem. 1843

13. Kolavi G, Hegde V and Khazi I 2006 Tetrahedron Lett. 472811

14. Kolavi G, Hegde V, Khazi I and Gadad P 2006 Bioorg. Med. Chem. 143069

15. Hegde V S, Kolavi G D and Khazi I M 2006 J. Sulfur Chem. 27307

16. Rosowsky A, Chaykovsky M, Chen K K N, Lin M and Modest E J 1973 J. Med. Chem. 16185

17. Dave C G and Shah R D 1998 J. Het. Chem. 351295

18. Review: Sebnis R W, Ragnekar D W and Sonawane N D 1999 J. Het. Chem. 36333

19. Gewald K, Schinke E and Boettcher H 1966 Chem. Ber. 9994

20. National Committee for Clinical Laboratory Standards 1996 Method for broth dilution antifungal susceptibility testing yeast. Approved standard, M27-A, 15, 10, NCCLS, VA Medical Center, Tuscon

21. National Committee for Clinical Laboratory Standards 2002 Methods for dilution antimicrobial susceptibility tests for bacteria that grow aerobically. Third edn, Approved standard. NCCLS document M100-S12, NCCLS, Wayne, PA, USA 